\title{
Anatomia humana como ferramenta para promoção de educação em saúde na adolescência
}

\author{
Magna Carolina de Melo ${ }^{1}$, Jaqueline Gabriele Silva ${ }^{1}$, Caio Godinho Caldeira ${ }^{1}$, Luisa Machado dos \\ Santos Rocha ${ }^{1}$, Brenda Oliveira ${ }^{1}$, Sarah Tereza Siqueira ${ }^{1}$, Luis Gustavo de Almeida Ribeiro ${ }^{2}$, Luciana \\ Estefani Drumond de Carvalho ${ }^{3}$
}

Resumo: A adolescência é um período de transição, marcado por transformações psicossociais, biológicas e comportamentais. Conhecer e compreender o corpo humano previne danos à saúde nessa fase. O projeto "Uma visita ao corpo humano", desenvolvido na Universidade Federal de São João delRei, campus Centro-Oeste (UFSJ/CCO), baseou-se em visitas ao laboratório de Anatomia Humana, a fim de oferecer aos adolescentes do ensino médio e fundamental II das escolas públicas e privadas de Divinópolis, Minas Gerais, um esclarecimento acerca dos sistemas do corpo, enfatizando a saúde e o autocuidado. Foram selecionados 6 sistemas para as discussões, para as quais utilizaram-se peças sintéticas. Durante o ano de 2017, realizaram-se 18 visitas de 15 escolas diferentes. Foram atendidos 721 alunos. Desses, 350 (48,5\%) frequentadores da rede pública, e 371 (51,5\%) da particular. Do total, 458 foram alunos $(63,5 \%)$ do ensino fundamental e 263 (36,5\%) do ensino médio. Os resultados foram positivos, visto que os estudantes obtiveram mais acertos nas perguntas do teste, após as discussões. Além disso, observou-se superioridade do conhecimento prévio dos estudantes do ensino fundamental de escolas privadas.

Palavras-chave: Adolescentes; Autocuidado; Corpo Humano; Extensão Universitária; Ensino Básico

\section{Human anatomy as a tool to promote health education in adolescence}

Abstract: Adolescence is a period of transition marked by psychosocial, biological, and behavioral changes. Knowing and understanding the human body prevents harm during this phase. The project "A visit to the human body" ("Uma visita ao corpo humano") developed at the Federal University of São João del-Rei, campus Centro-Oeste (UFSJ / CCO), was based on visits to the human anatomy laboratory, in order to offer high school and elementary school adolescents from public and private schools in Divinópolis, Minas Gerais State, Brazil, an explanation about the systems of the body, emphasizing health and self-care. Six systems were selected for the discussions, in which synthetic parts were used. During 2017, 18 visits were made from 15 different schools, and 721 students were served. Of these, 350 $(48.5 \%)$ attended public schools and 371 (51.5\%), private ones. Of the total, 458 (63.5\%) of elementary school and 263 (36.5\%) of high school. The results were positive since the students scored better after the discussions. Moreover, higher knowledge was observed in primary school students from private schools.

Keywords: Adolescents; Selfcare; Human Body; Health Education; University Extension; Elementary School

\author{
Originais recebidos em \\ 07 de abril de 2020 \\ Aceito para publicação em \\ 14 de setembro de 2020 \\ Discente do curso de \\ Medicina da Universidade \\ Federal de São João del-Rei \\ (UFS) campus Centro-Oeste
}

1

\section{2}

Discente do curso de Bioquímica da Universidade Federal de São João del-Rei (UFSJ) campus Centro-Oeste

3

Docente do curso de Medicina da Universidade Federal de São João del-Rei (UFSJ) campus Centro-Oeste

lucianadrumond@ufsj.edu.br (autora para correspondência) 


\section{Introdução}

A pré-adolescência e a adolescência são períodos de transição entre a infância e a vida adulta, que vão dos 10 aos 19 anos (Organização Mundial de Saúde, 2014), caracterizados por uma série de mudanças e transformações, tanto no aspecto psicossocial, quanto no biológico e comportamental. Nessa fase, os indivíduos começam a se relacionar com o ambiente ao seu redor de forma mais independente, e por se encontrarem em situação de vulnerabilidade individual e social, acabam sendo expostos a riscos, como uso de drogas lícitas e ilícitas, gravidez indesejada, e a doenças sexualmente transmissíveis (Oliveira et al., 2020).

O adolescente em condição peculiar de desenvolvimento, demanda atenção e cuidado integral, uma vez que, nessa fase, se estabelecem hábitos e atitudes que irão repercutir na vida adulta. Neste contexto, as medidas de educação em saúde assumem papel fundamental na prevenção de danos à saúde, através de incentivos e orientações, visando conscientizar o adolescente a se tornar sujeito ativo nos seus cuidados (Carvalho et al., 2020).

Considerando que a adolescência é um período marcado por transformações biológicas, conhecer os sistemas do corpo humano e seu funcionamento, a partir do ensino da anatomia humana, contribui positivamente para a melhor compreensão das mudanças que ocorrem no corpo durante essa fase (Baptista et al., 2015).

A disciplina de Biologia nas escolas contempla diversos conteúdos, entre eles o conhecimento do corpo humano, abrangendo noções sobre a anatomia humana, a fisiologia e sobre a saúde no geral. No entanto, em muitas dessas escolas o material didático disponível se restringe aos livros, que certamente consiste em um recurso de grande importância, porém não deve ser o único (Baptista et al., 2015).

Sabe-se que, para compreender adequadamente a teoria, é preciso experienciá-la (Russi, 2011). Assim, os conteúdos teóricos, quando associados à prática, ampliam a criatividade e interação do aluno, resultando em uma participação mais ativa no processo educativo, com melhor aproveitamento e assimilação do conteúdo ensinado (Filadelfi et al., 2015).

A aproximação entre teoria e prática do presente trabalho, busca oferecer uma visão geral dos sistemas do corpo humano para os adolescentes do ensino médio e fundamental II das escolas púbicas e privadas do município de Divinópolis-MG, através de visitas guiadas ao laboratório de Anatomia Humana da Universidade Federal de São João del Rei, Campus Centro-Oeste (UFSJ/CCO. Divinópolis é uma cidade com população estimada de 238.230 habitantes, contando com 86 estabelecimentos de ensino fundamental e 28 estabelecimentos de ensino médio (Instituto Brasileiro de Geografia e Estatística, [IBGE], 2017). O projeto foi divulgado e as visitas foram disponibilizadas para todas as escolas que fizeram o contato e solicitaram marcação.

Este projeto de extensão oferece aos adolescentes oportunidade de integrar o conhecimento teórico adquirido em sala de aula com a prática, através de atividades teórico-práticas, nas quais são utilizadas peças sintéticas, representando de forma mais realística os sistemas do corpo humano, tornando o aprendizado mais dinâmico e o conteúdo menos abstrato. Contemplamos aqui o desenvolvimento de atividades envolvendo a participação da comunidade, buscando, portanto, promover integração e transmitir o conhecimento (Silva et al., 2018).

As atividades abordam conteúdos de anatomia humana que, correlacionados com a fisiologia, trazem como enfoque principal as doenças relacionadas a cada sistema, ressaltando a importância dos cuidados com a saúde, as medidas de prevenção e incentivando o autocuidado.

Além disso, as visitas propiciam uma troca de conhecimento e experiência entre estudantes universitários e os visitantes das escolas participantes. Contempla-se, também, maior aproximação da universidade com a 
comunidade na qual está inserida, visando, de forma objetiva e transparente, uma integração dos alunos visitantes com o ambiente universitário.

Neste contexto, o projeto de extensão "Uma visita ao corpo humano", cujos dados foram utilizados para a escrita deste artigo, teve como objetivo oportunizar discussão sobre saúde entre os adolescentes, descrevendo a função geral e os componentes dos principais sistemas do corpo humano, com ênfase em noções básicas de saúde e promoção do autocuidado.

\section{Métodos}

As atividades de extensão foram desenvolvidas no período de 2016 a 2017 por acadêmicos dos cursos de medicina, enfermagem, farmácia e bioquímica da Universidade Federal de São João del Rei - Campus CentroOeste (UFSJ-CCO), situado em Divinópolis - MG, sob orientação e supervisão da docente responsável.

Os aspectos éticos do projeto seguem as orientações da resolução № 510, de 07 de abril de 2016 do Conselho Nacional de Saúde, parágrafo único - item V, que especifica que não serão registradas nem avaliadas pelo sistema CEP/CONEP pesquisa com bancos de dados, cujas informações são agregadas, sem possibilidade de identificação individual.

Os extensionistas envolvidos no projeto foram escolhidos por meio de processo seletivo, devidamente capacitados para lecionar anatomia teórica, prática e sua aplicação no cotidiano. Foram realizadas também reuniões para treinamento dos alunos extensionistas, aperfeiçoamento e discussão acerca do projeto. Com o intuito de padronizar as atividades preparadas por diferentes alunos e fornecer as informações com embasamento científico, foram confeccionados e revisados, pelos extensionistas e pela coordenadora do projeto, materiais didáticos (roteiros) adequadamente referenciados sobre cada tema, revisando anatomia, fisiologia, e doenças relacionadas a cada sistema. Os sistemas do corpo humano selecionados para abordagem foram: digestório, cardiovascular, urinário, respiratório, genital feminino e masculino.

O público do projeto foi constituído de adolescentes do ensino fundamental II e ensino médio de escolas públicas e privadas do município de Divinópolis - MG. As aulas aconteceram no Laboratório de Anatomia Humana da UFSJ-CCO.

O projeto contou com divulgação nas redes sociais e por e-mail das escolas. A participação ocorreu sob demanda. $O$ agendamento das escolas que responderam ao contato da equipe e manifestaram interesse foi feito via e-mail, após confirmação da disponibilidade do Laboratório de Anatomia Humana da UFSJ-CCO e dos alunos extensionistas para a data requisitada. Foram enviados, então, três documentos: a carta convite, o termo de compromisso e as orientações gerais para a entrada no laboratório. Cada visita podia contar com, no máximo, 60 alunos, que se dividiam em dois grupos de 30 alunos em cada laboratório, sendo que cada grupo era acompanhado por um responsável da escola visitante. Para introduzir a visita, foi realizada uma conversa com os alunos sobre a Universidade, os cursos oferecidos no campus, ressaltando o caráter público da instituição.

Para avaliação prévia dos estudantes visitantes sobre os conhecimentos do corpo humano, foram aplicados testes apropriados a cada nível educacional. Após a visita, foram aplicados novos testes com as mesmas questões e com uma subsequente discussão.

Para o ensino fundamental, foi aplicada atividade composta por 34 itens, contendo um conjunto de imagens para identificação dos componentes de cada sistema e do posicionamento anatômico adequado das estruturas. Além disso, a atividade continha uma questão dissertativa referente ao sistema e à doença relacionada. Desta forma, cada grupo poderia obter uma pontuação máxima de 34 pontos. 
Já para o ensino médio, foi elaborado um questionário composto por 18 questões de múltipla escolha, abordando os sistemas apresentados, hábitos de vida e doenças associadas. Desta forma, cada grupo poderia obter uma pontuação máxima de 18 pontos.

Os testes eram, então, distribuídos aos alunos, que se organizavam em grupos de quatro pessoas, para resolução das questões em um tempo de 15 minutos, com o objetivo de avaliar seus conhecimentos prévios à aula expositiva. Em seguida, dava-se início às discussões sobre o corpo humano, usando as peças sintéticas do laboratório. Ao final, eram apresentadas algumas peças naturais disponíveis no laboratório. Então, o mesmo teste era aplicado novamente. O responsável pela escola visitante também recebia um questionário, que visava avaliar a visita com observações, críticas e sugestões, com relação ao projeto de extensão.

Periodicamente, os extensionistas se reuniam com a docente responsável para capacitação continuada e discussão de possíveis melhorias indicadas pelos visitantes, e observadas pela vivência dos próprios extensionistas. A experiência com as visitas forneceu aos extensionistas informações importantes, que se tornaram pautas de discussões nas reuniões semanais, e resultaram em readequações e melhorias do projeto.

Além de uma análise descritiva da amostra, mensurou-se a efetividade da intervenção educativa do projeto comparando-se o número de acertos nas atividades antes e depois das discussões com os extensionistas. $\mathrm{O}$ número amostral se refere a grupos, compostos por 4 a 6 alunos. Quanto à análise, os dados coletados foram armazenados e convertidos em planilhas no programa Microsoft Excel 2010. A análise estatística foi realizada com o auxílio do software GhaphPad Prism, onde foram confeccionados os gráficos. Para verificar a distribuição da amostra, foi utilizado o teste de D'Agostino e Pearson. Como as variáveis se mostraram paramétricas, os dados foram descritos como média de acertos nas atividades \pm erro padrão da média. Foram consideradas duas variáveis independentes: o tipo de escola (pública ou privada) e o tempo de aplicação do teste (antes ou depois). Os testes estatísticos utilizados foram o ANOVA de dois fatores e o pósteste de Tukey. A diferença entre os valores foi considerada estatisticamente significativa quando $p<0,05$ (intervalo de confiança de 95\%).

\section{Resultados}

Durante o ano de 2017 foram atendidos 721 alunos, em 18 visitas. Desses, 350 (48,5\%) alunos eram frequentadores da rede pública de ensino e 371 (51,5\%) alunos de escolas particulares. Do total, 458 (63,5\%) eram alunos do ensino fundamental e 263 (36,5\%) alunos do ensino médio.

Os valores obtidos nos testes para as escolas públicas ( $n=26$ grupos) foi de $25,1 \pm 1,5$ acertos antes e $30,5 \pm 0,7$ acertos após $(p<0,05)$. Já para as instituições privadas ( $n=16$ grupos), os valores foram $30,7 \pm 0,8$ acertos antes e 33,0 $\pm 0,3$ acertos após $(p>0,05)$, (Figura 1). Em relação ao ensino fundamental, observa-se diferença nos valores iniciais de acertos entre as instituições públicas $(25,1 \pm 1,5)$ e privadas $(30,7 \pm 0,8)(p<0,05)$. Além disso, os estudantes de escolas públicas obtiveram maior número de acertos no teste aplicado após a discussão dos sistemas, em relação ao teste inicial. Entretanto, para os estudantes de escolas privadas não se observou diferença significativa no número de acertos no teste aplicado, antes e após a discussão dos sistemas.

Para o ensino médio, o número de acertos após a visita e discussão foi maior do que antes, para as duas categorias de escolas. Os valores obtidos para escolas públicas ( $n=11$ grupos) foram $15,1 \pm 0,4$ acertos antes e $16,9 \pm 0,3$ acertos após $(p<0,05)$. Já para as instituições privadas ( $n=22$ grupos), os valores foram $15,7 \pm 0,2$

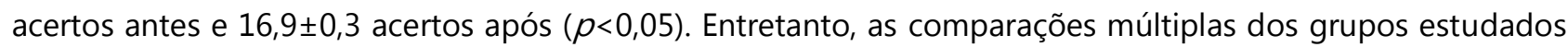
não permitiram detectar diferenças nos valores de acertos entre as instituições públicas e privadas (Figura 2). 


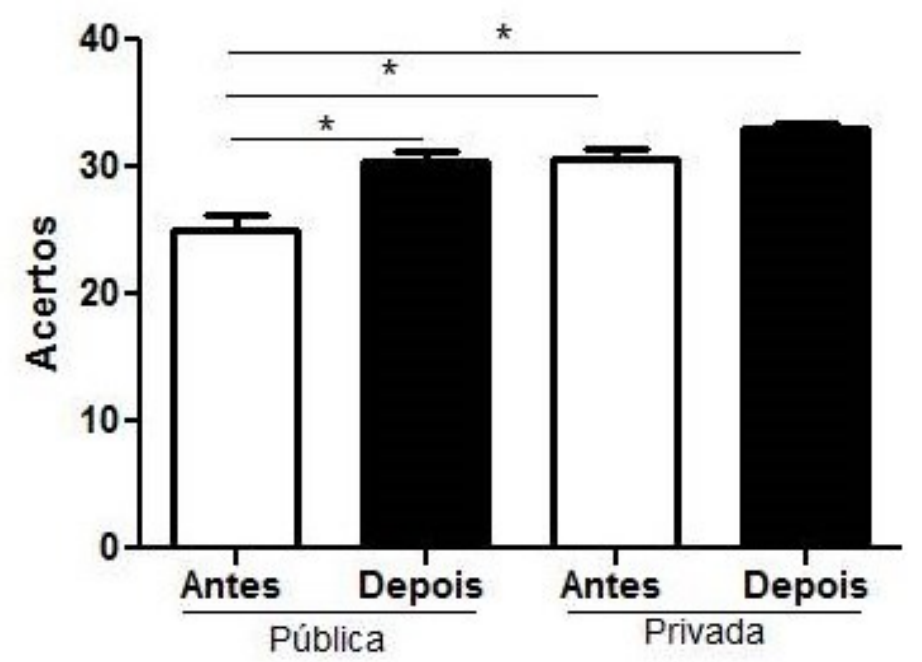

Figura 1. Desempenho no ensino fundamental. Divinópolis - MG. 2017. Comparação entre escolas públicas e privadas, nos tempos antes e depois, através do teste ANOVA de dois fatores e pós-teste de Tukey. Os dados estão representados como média \pm EPM. $\left({ }^{*} p<0,05\right)$. Fonte: autores.

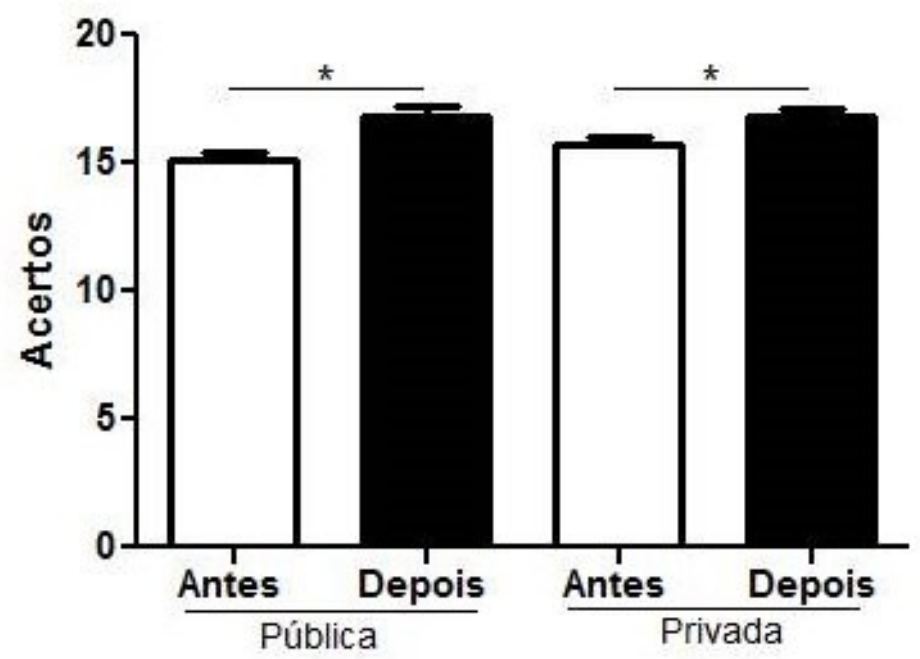

Figura 2. Desempenho no ensino médio. Divinópolis - MG. 2017. Comparação entre escolas públicas e privadas, nos tempos antes e depois, através do teste ANOVA de dois fatores e pós-teste de Tukey. Os dados estão representados como média \pm EPM. $\left({ }^{*} p<0,05\right)$. Fonte: autores.

\section{Discussão}

No transcorrer deste trabalho de educação em saúde, os estudantes universitários extensionistas tiveram uma participação ativa, fato muito favorável para o aprimoramento de seus conhecimentos. $O$ contato do acadêmico com a sociedade beneficiada foi extremamente positivo para o seu desenvolvimento, tendo em vista que a absorção do conteúdo teórico é mais intensa quando esse é utilizado na gratificante interação com o público geral (Silva et al., 2018).

O número expressivo de alunos atendidos durante o ano de 2017, totalizando 721 escolares, reflete a grande procura pelo projeto. Destaca-se que essa busca significativamente elevada se justifica devido à carência do ensino prático nas escolas, consequência da ausência de laboratórios, equipamentos e recursos didáticos apropriados. Ademais, a escassez de métodos teóricos cativantes de ensino com temas pertinentes ao corpo 
humano, torna os recursos do laboratório universitário ainda mais interessantes e instigadores, favorecendo a alta demanda (Cavalcanti et al., 2020).

Neste trabalho tivemos uma prevalência de atendimento aos alunos do ensino fundamental, sendo $63,5 \%$ do total de atendimentos, fato condizente com o perfil escolar característico de várias cidades brasileiras. Esses números evidenciam o considerável processo de evasão escolar no ensino médio, problema marcante no Brasil, observado até em países com alto desenvolvimento econômico (Soares et al., 2015).

O aumento do número de acertos nas questões, quando comparados antes e após as discussões com os extensionistas, tanto para o ensino fundamental quanto para o médio, revelam a efetividade da intervenção proposta pelo projeto, indicando melhora no conhecimento, ainda que a curto prazo, sobre os conteúdos de saúde abordados. Apenas para os estudantes de escolas privadas do segmento fundamental, não se observou aumento no número de acertos após a visita. Acredita-se que as questões propostas pelo projeto podem ter se apresentado com baixo grau de dificuldade para os estudantes, uma vez que muitos grupos obtiveram nota máxima, mesmo antes de iniciar as visitas. Desta forma, seria importante reanalisar o instrumento de avaliação.

Dentre os resultados observados, nota-se um melhor desempenho dos escolares oriundos do ensino fundamental privado, que obtiveram mais acertos nas questões propostas antes do início das discussões, revelando superioridade em termos de conhecimento prévio. Essa diferença vai ao encontro da literatura científica, corroborando com a disparidade de desempenho usualmente observada entre escolas privadas e públicas, em que as primeiras apresentam, majoritariamente, melhores índices (de Moraes \& Belluzzo, 2014).

Outro achado relevante é o aumento do número de acertos dos alunos de escolas públicas após explanação dos extensionistas. A evolução observada é condizente com os dados da literatura, em que em projeto semelhante desenvolvido com 31 participantes, também se observou melhora de aproveitamento nos questionários aplicados após a exposição, sendo a média total de acertos de $54,2 \%$ no primeiro teste e de $82,3 \%$, no segundo (Maturana \& Costa, 2013). Além disso, a melhoria observada confirma a efetividade das aulas ministradas, reforçando a premissa de que a capacidade de seleção e retenção de informações é aperfeiçoada com o uso de atividades práticas (Zanesco et al., 2017). É importante ressaltar que a técnica utilizada de testes preliminares e posteriores não tem abrangência suficiente para medir a retenção do aprendizado, já que a avaliação acontece logo após o treinamento. Entretanto, essa forma permite avaliar a evolução do aluno após a experiência didática, a efetividade do treinamento e a capacidade dos participantes de absorver as novas informações apresentadas durante o projeto (Sefer et al., 2019). São necessários estudos posteriores para avaliação do impacto a longo prazo.

Dentre os pontos fortes deste projeto, destaca-se a sua replicabilidade, tendo em vista que pode ser implementado em outras universidades dotadas de laboratórios de anatomia. Além disso, a relevância do tema propicia a aproximação do conhecimento científico e cotidiano, capaz de despertar o interesse dos alunos em relação à universidade e ao ambiente acadêmico (Cavalcanti et al., 2020). Dessa forma, os resultados positivos identificados nos escolares envolvidos no projeto podem ser expandidos para outros estudantes.

\section{Conclusão}

As visitas guiadas ao laboratório de anatomia permitiram complementar o conteúdo de anatomia e fisiologia humana, aprendidos em sala de aula, bem como contribuíram para a melhor compreensão dos sistemas do corpo humano. Tais conhecimentos conferem aos adolescentes mais autonomia e consciência em seus cuidados com a saúde. Além disso, o contato direto com a Universidade pode motivar os alunos a permanecerem no meio acadêmico após concluírem o ensino médio.

Assim, este projeto possibilitou experiências didáticas inovadoras, em educação formal e não formal, para 
estudantes e professores das escolas de Divinópolis, contribuindo também para a melhoria do ensino de ciências por meio da complementação com os recursos humanos e materiais disponíveis na universidade.

Essa experiência contribuiu também com a formação didática dos extensionistas acadêmicos da UFSJ, que tiveram a oportunidade de aperfeiçoar seus conhecimentos acerca de conteúdos importantes na formação de profissionais da área da saúde.

\section{Agradecimentos}

À Universidade Federal de São João del Rei, campus Centro-Oeste, às escolas de ensino público e privado do município de Divinópolis-MG; ao apoio da Pró-Reitoria de Extensão e Assuntos Comunitários - PROEX. Ao apoio técnico de Marina Paula da Cunha Oliveira.

\section{Contribuição de cada autor}

M.C.M. (bolsista PIBEX/UFSJ/CCO) gerenciou as atividades realizadas no desenvolvimento do trabalho, escreveu o texto final, preparou e ministrou as aulas de anatomia para os alunos do ensino fundamental e médio. S.T.S e L.G.A.R. prepararam e ministraram as aulas de anatomia para os alunos do ensino fundamental e médio e participaram da escrita do texto final. C.G.C. e L.M.S.R. prepararam e ministraram as aulas de anatomia para os alunos do ensino fundamental e médio e contribuíram para a escrita e revisão do texto final. B.O. e J.G.S. prepararam e ministraram as aulas de anatomia para os alunos do ensino fundamental e médio e contribuíram para a escrita e revisão do texto final. Colaborou com a análise estatística e revisão do texto final, L.E.D.C., atuou como coordenadora e orientadora dos bolsistas e voluntários, além da orientação da escrita do artigocientífico.

\section{Referências}

Baptista, V. I. D. A., Lima, J. de M., Medeiros, L. M. de A., Scardua, A., \& Baptista, J. da S. (2015). Concepções sobre anatomia humana de alunos do ensino médio da cidade de Cuité-PB: Funções e relações com cotidiano Revista Brasileira de Pesquisa Em Educação Em Ciências, 15(1), 59-78.

Carvalho, H. D. G., Lira, J. M. C., Lessa, A. L., dos Santos, V. F., Deda, A. V., Rodrigues, L. M. C. L., ... \& Fraga, B. P. (2020). Atividade lúdico-educativa para ensino de neurociência aos escolares da rede pública. Brazilian Journal of Health Review, 3(3), 6458-6466.

Cavalcanti R. S., Rodrigues L. M. C. L., Albuquerque U. M. L. A. C., Nascimento J., Filho M. T. B., Santana M. F. S., ..., \& Garção D. C. (2020). O Ensino de anatomia humana em escolas públicas de Sergipe como projeto de extensão universitária. Brazilian Journal of Development, 6(7), 45974-45986.

de Moraes, A. G. E., \& Belluzzo, W. (2014). O diferencial de desempenho escolar entre escolas públicas e privadas no Brasil. Nova Economia, 24(2), 409-430.

Instituto Brasileiro de Geografia e Estatística (2017). Cidades. [S. I.]: IBGE. Recuperado de https://cidades.ibge.gov.br/ $\underline{\mathrm{brasil} / \mathrm{mg} / \text { divinopolis/panorama }}$

Filadelfi, A. M. C., Carvalho, L. B., Nascimento, V. S., Tobaldini, G., \& Conceição, L. T. (2015). Instrumentos pedagógicos interativos no ensino de Fisiologia e noções de saúde para jovens. Revista Brasileira de Extensão Universitária, 6(1), 1524.

Maturana, L. G., \& Costa, J. S. R. (2013). Anatomia humana como proposta prático-pedagógica para aplicar o tema transversal saúde na rede estadual de ensino de Diamantina - MG. Revista Vozes dos Vales da UFVJM: Publicações Acadêmicas, 3(2), 1-13. 
Oliveira P. C., Reis M. L., Vandenberghe L., Souza M. M., \& Medeiros M. (2020). “Sobrevivendo”: Vulnerabilidade social vivenciada por adolescentes em uma periferia urbana. Interface (Botucatu), 24: e190813.

Organização Mundial da Saúde (2014). Saúde para os adolescentes do mundo: Uma segunda chance na segunda década. Genebra: OMS.

Russi, A. C. R. (2011). Freire, Paulo. Pedagogia da autonomia: saberes necessários à prática educativa. 39. ed. São Paulo: Paz e Terra, 2009. Revista Da Faculdade de Educação Física da UNICAMP, 9(2), 219-226.

Sefer, C. C. I., Tavares, L. F., Silva, R. de A., Portella, M. B., \& Botelho, N. M. (2019). Avaliação do desempenho de estudantes de Medicina em Neurologia utilizando metodologias ativas. Revista Eletrônica Acervo Saúde, 11(9), e293.

Silva, J. H., Foureaux, G., Sá, M. A. de, Schetino, L. P. L., \& Guerra, L. B. (2018). O ensino-aprendizagem da anatomia humana: Avaliação do desempenho dos alunos após a utilização de mapas conceituais como uma estratégia pedagógica. Ciência \& Educação (Bauru), 24(1), 95-110.

Soares, T. M., Fernandes, N. da S., Nóbrega, M. C., \& Nicolella, A. C. (2015). Fatores associados ao abandono escolar no ensino médio público de Minas Gerais. Educação e Pesquisa, 41(3), 757-772.

Zanesco, C., da Silva, A. M., de Resende, D. T., \& Bagatini, M. D. (2017). Ensino de anatomia humana: experiência de integração da extensão universitária com ensino médio. Revista Ciência em Extensão, 13(3), 127-135.

\footnotetext{
Como citar este artigo:

Melo, M. C. de, Silva, J. G., Caldeira, C. G., Rocha, L. M. dos S., Oliveira, B., Siqueira, S. T., Ribeiro, L. G. de A., \& Carvalho, L. E. D. de. (2020). Anatomia humana como ferramenta para promoção de educação em saúde na adolescência. Revista Brasileira de Extensão Universitária, 11(3), 331-338. https://periodicos.uffs.edu.br/index.php/RBEU/article/view/11447/pdf
} 